\title{
Opportunistic Channel Access Scheme for Cognitive Radio System Based on Residual White Space Distribution
}

\author{
Manuj Sharma and Anirudha Sahoo \\ Indian Institute of Technology Bombay, Mumbai, India \\ Email: \{manuj, sahoo\}@it.iitb.ac.in
}

\begin{abstract}
We propose an opportunistic channel access scheme for cognitive radio-enabled secondary networks. In our work, we model the channel occupancy due to Primary User (PU) activity as a 2-state Alternating Renewal Process, with alternating busy and idle periods. Once a Secondary Node (SN) senses the channel idle, the proposed scheme uses the residual idle time distribution to estimate the transmission duration in the remaining idle time, subject to an acceptable PU interference constraint. The $\mathrm{SN}$ transmits the frames within the transmission duration without further sensing the channel, thereby reducing the average sensing overhead per transmitted frame. The analytical formulation used by the scheme does not require the $\mathrm{SN}$ to keep track of the start of the idle period. We validate the analytical formulations using simulations, and compare the performance of the proposed scheme with a Listen-Before-Talk (LBT) scheme.
\end{abstract}

\section{INTRODUCTION}

Opportunistic Spectrum Access (OSA) has emerged as a promising approach to efficiently utilize the electromagnetic spectrum. In this approach, the secondary users use those parts of the spectrum band that are not currently utilized in space or time by any primary user. Channel occupancy (channel idle and busy time) distribution due to Primary User (PU) activity has recently been used to devise opportunistic channel access schemes for secondary networks. Analytical formulation of most of these schemes assume that either the start of idle period (white space ${ }^{1}$ ) is known to the Secondary Node (SN), or the secondary network transmission is slotted and the SN senses the channel at the beginning of each slot. In this paper, we propose an opportunistic channel access scheme in which a secondary node senses the channel only when it has one or more frames to transmit. If the channel is sensed idle, it uses the residual idle time distribution to estimate its transmission duration within the remaining idle time, without requiring the knowledge of start of the idle period. Within the estimated transmission duration, the SN transmit the frames without further sensing the channel, thereby reducing the average sensing overhead per secondary frame transmission. The schemes, which assume knowledge of the start of the idle period, require the SN to sense the channel continuously to keep track of the start of each idle period, which is costly for energy-constrained and mobile SNs. As an example, in Figure 1, if an SN transmits the first burst of frames between time

\footnotetext{
${ }^{1}$ In this paper, we use the terms channel idle time/white space and channel residual idle time/residual white space synonymously.
}

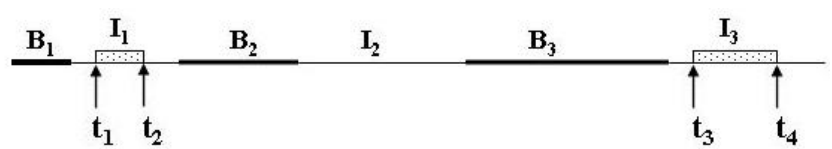

Fig. 1: Alternating Renewal Process Model for PU Channel Occupancy

instants $t_{1}$ and $t_{2}$ in idle period $I_{1}$, and the second burst arrives at time $t_{3}$ in idle period $I_{3}$, then such schemes requires the $\mathrm{SN}$ to continuously sense the channel between instants $t_{2}$ and $t_{3}$ so as to keep track of the start of idle period $I_{3}$. On the other hand, our proposed scheme does not require continuous sensing of the channel and performs channel sensing only at time instants $t_{1}$ and $t_{3}$ when frames arrive at the MAC layer of SN. But in absence of any knowledge of the idle cycle start time, it has to estimate its transmission duration solely based on the remaining idle time distribution of the channel. Both the approaches usually assume the knowledge of the channel occupancy distribution, obtained by some appropriate means.

\section{RELATED WORK}

Partially-Observed Markov Decision Process (POMDP) framework has been used in [1] and [2] to propose channel sensing and transmission strategies, under the assumption of slotted Primary and Secondary networks. This assumption requires synchronization between the Primary and Secondary transmission slot structures. In [3] and [4], the authors consider an unslotted Primary Network with multiple channels and a slotted Secondary Network, which sense the channels at the beginning of each slot. In [3], an optimal joint sensing and access strategy for the secondary networks is proposed. In [4], the authors have proposed a learning-based approach to maintain and use belief vectors for channel access.

The authors in [5] approximated a Semi-Markov model for WLAN channels with continuous-time Markov chain and proposed the Cognitive Medium Access (CMA) protocol for slotted secondary network. In this protocol, the SN sense the channels at the beginning of every slot, and cast the channel access problem as constrained Markov decision process (CMDP) for fully observable system. In [6], authors consider an unslotted Primary network, but their analytical formulation of the access scheme is based on the assumption that the SN can detect the beginning of the idle period. A proactive spectrum access approach based on a three-tier 
predictive statistical model of spectrum availability is proposed in [7]. In [8], the authors have used PDF and CDF of a whitespace trace (idle periods), obtained using simulation of a linear array of five primary WLAN nodes, to compute the sensing duration and the number of frames that an SN should transmit on sensing a channel idle, subject to a PU interference bound. The paper computes, both analytically and using simulations, the Effective Secondary Throughput, and Primary User Interference, but it assumes that the start of idle period is known.

Contrary to the above mentioned schemes, our scheme does not make any assumption regarding the transmission structure of primary and secondary networks and is based on the residual idle time distribution of the channel. Once a secondary node senses the channel idle, it estimates, using the theory proposed in this paper, the transmission duration in the remaining white space so that the PU interference bound is not violated. It then transmits appropriate number of frames within the estimated duration.

\section{System ModeL}

We consider a spectrum sharing model in a wireless network. The network communicates using a single channel. The designated users of the single channel are set of users termed as Primary Users (PU) of the channel. There are other set of nodes known as Secondary Nodes (SN) which share the channel with PUs. However, the SNs can only access the channel when no PU is using the channel. Thus, the $\mathrm{SNs}$ have to look for the so called white spaces (i.e., idle periods in the channel) and opportunistically transmit their packets. The PUs are not aware of secondary node's transmission and can initiate their transmission whenever they require. It is secondary node's responsibility to detect the PU transmission and evacuate the channel. So, the SNs must ensure that their transmissions do not cause interference to any PU beyond a certain limit. This limit is specified by the PU which we denote as $\eta$. Thus, $\eta$ is the upper bound of probability of interference by SNs which PUs can tolerate. Lower/higher value of $\eta$ indicates lower/higher tolerance of PU to SN-generated interference. Our model does not assume any specific MAC protocol or transmission structure (slotted/unslotted) for the PU. Though in this work, we have considered a single pair of secondary sender and receiver nodes which opportunistically use the channel, the scheme can be used with multiple pairs of secondary sender and receiver nodes, provided the secondary nodes use a standard MAC protocol to coordinate channel access among them.

From a secondary node's perspective, the channel is considered idle when it is not used by a PU and is considered busy if it is used by a PU. We model the channel occupancy due to PU activity as an Alternating Renewal Process (See Figure 1), in which a cycle consisting of channel busy duration (denoted as $B$ ) followed by channel idle duration (denoted as $I$ ), repeats (renews) in time. In this work we assume that the SN knows the channel idle and busy time distributions. Some of the earlier research work have also used this assumption

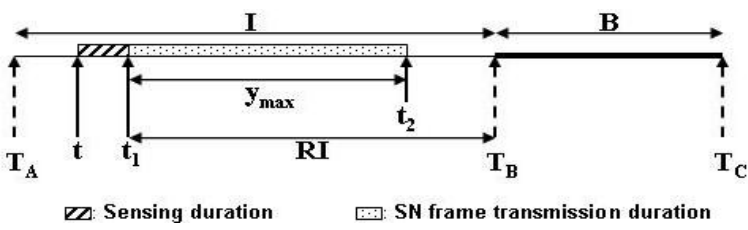

Fig. 2: Successful SN Transmission in the Residual Idle Time

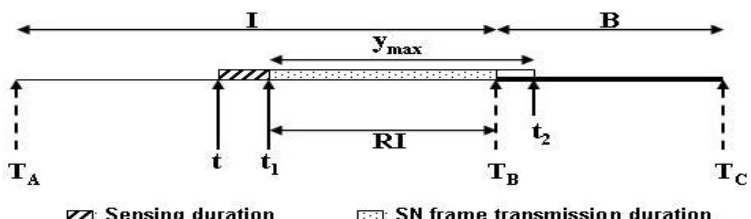

Fig. 3: Interference in PU Transmission due to SN Transmission

(see, for e.g., [1], [2], [3], [4], and [5]). This information can be made available to a secondary node by some designated central server, which measures the channel occupancy due to PU activity, fits the appropriate distribution and estimates the channel busy and idle time distribution parameters.

\section{OVERVIEW OF OpPortUNiSTIC SPECTRUM ACCESS USING RESIDUAL White SPACE DistribUtion}

Whenever a Secondary Node's MAC layer receives one or more packets for transmission, the node senses the channel. If the channel is sensed idle, the node concludes the presence of white space which can be used for secondary frames transmission. But in absence of continuous sensing, the node cannot keep track of the start of the white space (idle period). This sensing operation can be seen as random incidence in the idle cycle (such as at time instants $t_{1}$ and $t_{3}$ in Figure 1 and instants $t$ in Figure 2 and 3). In our work, we derive the residual white space distribution (or, residual idle time distribution) based on random incidence in a renewal cycle (see [9, pp. 328-331]), as explained in Section V. The SN uses the residual white space distribution to estimate the maximum duration for which it can transmit (say, $y_{\max }$ ) within the remaining white space so as to satisfy the acceptable interference constraint set by the PU. The SN transmits the frames within the estimated transmission duration without any further sensing of the channel. It receives an acknowledgment from the receiver for each successfully received frame. Absence of acknowledgment for a transmitted frame indicates collision of the frame or its acknowledgment with PU transmission due to appearance of the PU on the channel. Figure 2 and Figure 3 shows two possible transmission scenarios. In both the figures, $T_{A}, T_{B}$, and $T_{c}$ denote respectively the start of an idle cycle (white space), end of the idle cycle (or, equivalently, start of the next busy cycle due to PU transmission), and end of the busy cycle. On receiving one or more frames for transmission at time instant $t$, the SN senses the channel from time $t$ to $t_{1}$, and since the channel is sensed idle, it transmit frames for a maximum duration of $y_{\max }$ (which is equal to $\left.t_{2}-t_{1}\right)^{2}$. The transmission duration $y_{\max }$ is computed by the $\mathrm{SN}$ using analytical formulations explained in Section V. The duration

\footnotetext{
${ }^{2}$ If $\mathrm{SN}$ does not have enough frames to consume the duration of $y_{\max }$, it stops after sending all the frames.
} 
$\left(T_{B}-t_{1}\right)$ (represented as $\left.R I\right)$ denotes the residual white space (or residual idle time). In Figure 2, since $R I$ is more than $y_{\max }$, the $S N$ successfully transmits for complete $y_{\max }$ duration. On the other hand, in Figure 3, since $R I$ is less than $y_{\max }$, the $\mathrm{SN}$ transmits only for $R I$ duration and collides with the next busy cycle due to PU transmission, thereby interfering with the PU transmission.

After transmission (for $y_{\max }$ duration in Figure 2 and for $R I$ duration in Figure 3), if the $\mathrm{SN}$ has more frames to transmit, it backs off randomly for a duration, which is exponentially distributed with mean channel busy time period and senses the channel again. This backoff and sensing operation is repeated until the $\mathrm{SN}$ again (randomly) lands in an idle cycle (white space) and transmit additional frames for $y_{\max }$ duration.

\section{Channel Access Scheme}

In this section, we present the analytical formulations for computing maximum transmission duration for which an SN can transmit when it senses the channel to be idle. In first three subsections below, we present the theory for general channel idle time distribution, followed by application of this theory for two specific channel idle time distribution configurations.

\section{A. Access Strategy}

On detecting a white space, the $\mathrm{SN}$ needs to compute the duration for which it can transmit within the residual white space so as to satisfy the acceptable interference constraint specified by the PU. The SN uses the residual white space distribution to compute this transmission duration. The residual white space distribution is obtained using the known idle and busy time distributions for the channel.

Let $I$ and $B$ represent the random variables denoting channel idle and busy time values, and $F_{I}$ and $F_{B}$ represent respectively the channel idle and busy time distributions with known parameters. Let $f_{R I}$ represent the residual idle time (or residual white space) density function, which is computed by the $\mathrm{SN}$ as follows [9, pp. 331]:

$$
f_{R I}(y)=\frac{1-F_{I}(y)}{E[I]}
$$

The residual idle time distribution function can then be computed as follows:

$$
F_{R I}(y)=\int_{0}^{y} f_{R I}(z) \mathrm{d} z
$$

Here $E[I]$ denotes the mean channel idle time and $F_{R I}(y)$ denotes the probability that the residual white space is less than $y$. In other words, if $\mathrm{SN}$ transmits for duration $y$, then $F_{R I}(y)$ denotes the probability that the remaining white space will end (due to appearance of PU on the channel) before $\mathrm{SN}$ transmission is over. In this case, $\mathrm{SN}$ transmission will interfere with PU transmission. So, on sensing the channel idle, the SN computes the transmission duration $y$ in the remaining white space so that the following constraint is satisfied:

$$
F_{R I}(y) \leq \eta
$$

The maximum value of $y$ (which we denote as $y_{\max }$ ) for which the above inequality is satisfied, is taken as the transmission

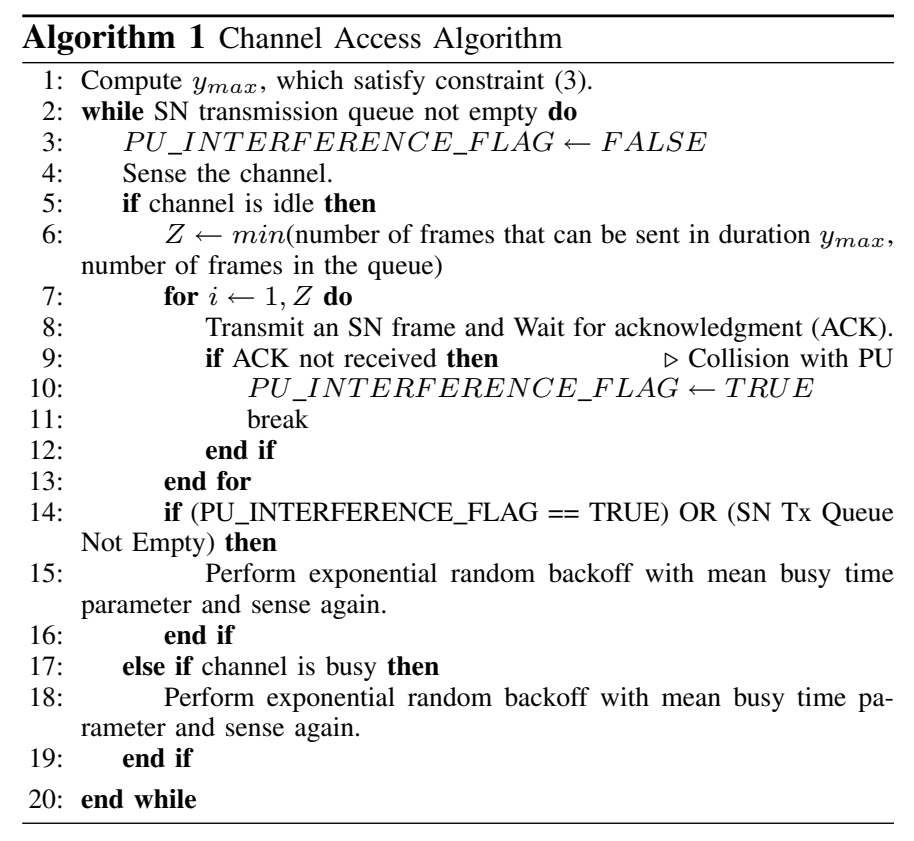

duration by the $\mathrm{SN}$ in the remaining white space. The channel access algorithm used by $\mathrm{SN}$ is broadly summarized in Algorithm 1.

For a given channel idle and busy time distribution, the value of $y_{\max }$ (computed using (3)) is fixed. Therefore, it can be computed by the SN once in the beginning (step 1 in Algorithm 1) and need not be computed every time the channel access is made. The frame transmission (step 8) within the for loop is performed without sensing the channel.

\section{B. Average Channel Utilization per White Space by Secondary Node}

There are two possible transmission scenarios (depicted in Figure 2 and Figure 3) for SN when it transmits for $y_{\max }$ duration on sensing the channel idle. In first scenario, if the remaining white space duration $(R I)$ is more than the transmission duration $y_{\max }$, then the channel is utilized for complete $y_{\max }$ duration and there is no interference to PU. In second scenario, if the remaining white space duration $(R I)$ is less than the transmission duration $y_{\max }$, then the channel is utilized only for $R I$ duration. In this scenario, the $\mathrm{SN}$ transmission interferes with PU transmission because the white space ends (due to PU's appearance on the channel) before the $\mathrm{SN}$ transmission is over. Therefore, for a given $y_{\max }$ value, the expression for average channel utilization per white space by the $\mathrm{SN}$ is given as:

$$
A U P W S=\frac{y_{\max }\left(1-F_{R I}\left(y_{\max }\right)\right)+\int_{q=0}^{y_{\max }} q f_{R I}(q) \mathrm{d} q}{E[I]}
$$

Here, integral variable $q$ denotes the values that residual idle time random variable $R I$ takes, and $E[I]$ is the mean idle time value. Note that $\left(1-F_{R I}\left(y_{\max }\right)\right)$ is the probability of successful SN transmission for $y_{\max }$ duration without interfering with PU. 
C. Primary User Interference Probability due to Secondary Transmissions

Primary User Interference Probability (PUIP) represents the probability that a secondary transmission interfere with PU transmission. The SN transmission interferes with the PU transmission if the actual residual white space turns out to be less than the transmission duration $y_{\max }$ computed by the SN using (3). Therefore, for the computed value of $y_{\max }$, the expression for $\mathrm{PU}$ interference probability $(P U I P)$ can be written as:

$$
P U I P=\int_{q=0}^{y_{\max }} f_{R I}(q) \mathrm{d} q
$$

where $f_{R I}$ is given by (1).

D. Formulations for 2-Erlang Distributed Channel Idle and Busy Time Values (Configuration-1)

In this configuration, channel idle and busy times are taken to be 2-Erlang distributed with parameters $\lambda_{i}$ and $\lambda_{b}$ respectively $\left(\lambda_{i}>0, \lambda_{b}>0\right)$. The idle time distribution function and the mean idle time value for 2-phase Erlang Distribution are given as:

$$
F_{I}(y)=1-e^{-\lambda_{i} y}\left[1+\lambda_{i} y\right], \quad y \geq 0
$$

and

$$
E[I]=\frac{2}{\lambda_{i}}
$$

Using the above equations and equations (1) and (2), we obtain the density and the distribution of residual idle time (refer to [10] for derivations):

$$
f_{R I}(y)=\frac{\lambda_{i} e^{-\lambda_{i} y}}{2}\left[1+\lambda_{i} y\right]
$$

and

$$
F_{R I}(y)=1-e^{-\lambda_{i} y}\left[1+\frac{\lambda_{i} y}{2}\right]
$$

Using equations (3) and (9), we obtain $y_{\max }$ by solving the following inequality for the maximum value of $y$ that satisfies the PU interference constraint:

$$
\begin{aligned}
1-e^{-\lambda_{i} y}\left[1+\frac{\lambda_{i} y}{2}\right] & \leq \eta \\
\Rightarrow e^{-\lambda_{i} y}\left[1+\frac{\lambda_{i} y}{2}\right]+(\eta-1) & \geq 0
\end{aligned}
$$

For the obtained value of $y_{\max }$, the expression for AUPWS can be written using equations (7), (8), (9) and (4) as follows (see [10] for derivations):

$$
A U P W S=\frac{\lambda_{i}}{2}\left[y_{\max } e^{-\lambda_{i} y_{\max }}\left(1+\frac{\lambda_{i} y_{\max }}{2}\right)+\int_{q=0}^{y_{\max }} q f_{R I}(q) \mathrm{d} q\right]
$$

Using (8), we solve the integral in the above expression as follows:

$$
\begin{aligned}
\int_{q=0}^{y_{\max }} q f_{R I}(q) \mathrm{d} q & =\int_{q=0}^{y_{\max }} \frac{1}{2} q \lambda_{i} e^{-\lambda_{i} q}\left[1+\lambda_{i} q\right] \mathrm{d} q \\
& =\frac{\lambda_{i}}{2} \int_{q=0}^{y_{\max }} q e^{-\lambda_{i} q} \mathrm{~d} q+\frac{\lambda_{i}^{2}}{2} \int_{q=0}^{y_{\max }} q^{2} e^{-\lambda_{i} q} \mathrm{~d} q
\end{aligned}
$$

Solving the integrals on the RHS of the above equation, we get,

$$
\int_{q=0}^{y_{\max }} q f_{R I}(q) \mathrm{d} q=\frac{3}{2 \lambda_{i}}-\frac{1}{2} \lambda_{i} e^{-\lambda_{i} y_{\max }}\left[y_{\max }^{2}+\frac{3 y_{\max }}{\lambda_{i}}+\frac{3}{\lambda_{i}^{2}}\right]
$$

Substituting the above integral value from (12) into (11), and solving the resultant expression, we get,

$$
A U P W S=\frac{\lambda_{i}}{2}\left[\frac{3}{2 \lambda_{i}}-e^{-\lambda_{i} y_{\max }}\left(\frac{y_{\max }}{2}+\frac{3}{2 \lambda_{i}}\right)\right]
$$

Similarly, for the obtained value of $y_{\max }$, the expressions for PUIP can be written using (8) and (5):

$$
\begin{aligned}
\text { PUIP } & =\int_{q=0}^{y_{\max }}\left(\frac{\lambda_{i} e^{-\lambda_{i} q}}{2}\left[1+\lambda_{i} q\right]\right) \mathrm{d} q \\
& =\frac{\lambda_{i}}{2} \int_{q=0}^{y_{\max }} e^{-\lambda_{i} q} \mathrm{~d} q+\frac{\lambda_{i}^{2}}{2} \int_{q=0}^{y_{\max }} q e^{-\lambda_{i} q} \mathrm{~d} q
\end{aligned}
$$

Solving the integrals on the RHS of the above equation, we get,

$$
P U I P=1-\frac{e^{-\lambda_{i} y_{\max }}}{2}\left(2+\lambda_{i} y_{\max }\right)
$$

\section{E. Formulations for Uniformly Distributed Channel Idle and Busy Time Values (Configuration-2)}

In this configuration, channel idle and busy time values are assumed to be Uniformly distributed between the positive values $(a, b)$, and $(c, d)$ respectively. The idle time distribution function and the mean idle time value for Uniform Distribution are given as:

$$
F_{I}(y)=\frac{y-a}{b-a}, \quad a \leq y<b
$$

and

$$
E[I]=\frac{a+b}{2}
$$

Using the above equations and equations (1) and (2), we obtain the density and distribution functions of residual idle time (refer to [10] for derivations):

and

$$
f_{R I}(y)=\frac{2(b-y)}{b^{2}-a^{2}}
$$

$$
F_{R I}(y)=\frac{\left(2 b y-y^{2}\right)}{b^{2}-a^{2}}
$$

Using equations (3) and (18), we obtain $y_{\max }$ by solving the following inequality for the maximum value of $y$ that satisfies 
TABLE I: SN Bursty Traffic Profile Parameters for Two Primary Channel Occupancy Configurations (all units in seconds)

\begin{tabular}{|c|cc|cc|cc|}
\cline { 2 - 7 } \multicolumn{1}{c|}{} & \multicolumn{2}{c|}{ Profile-1 } & \multicolumn{2}{c|}{ Profile-2 } & \multicolumn{2}{c|}{ Profile-3 } \\
\cline { 2 - 7 } \multicolumn{1}{c|}{} & $\mu_{O N}$ & $\mu_{O F F}$ & $\mu_{O N}$ & $\mu_{O F F}$ & $\mu_{O N}$ & $\mu_{O F F}$ \\
\hline Config-1 & 0.003 & 0.01 & 0.005 & 0.01 & 0.006 & 0.01 \\
\hline Config-2 & 0.2 & 0.5 & 0.3 & 0.5 & 0.4 & 0.5 \\
\hline
\end{tabular}

the PU interference constraint:

$$
\begin{aligned}
\frac{\left(2 b y-y^{2}\right)}{b^{2}-a^{2}} & \leq \eta \\
\Rightarrow y^{2}-2 b y+\eta\left(b^{2}-a^{2}\right) & \geq 0
\end{aligned}
$$

For the obtained value of $y_{\max }$, the expression for AUPWS can be written using equations (16), (17), (18) and (4) as follows (see [10] for derivations):

$$
\begin{aligned}
A U P W S= & \frac{2}{(a+b)}\left[\frac{y_{\max }\left\{\left(b^{2}-a^{2}\right)-2 b y_{\max }+y_{\max }^{2}\right\}}{\left(b^{2}-a^{2}\right)}\right. \\
& \left.+\int_{q=0}^{y_{\max }} \frac{2 q(b-q)}{b^{2}-a^{2}}\right]
\end{aligned}
$$

which can be solved to obtain

$A U P W S=\frac{2}{(a+b)\left(b^{2}-a^{2}\right)}\left[\left(b^{2}-a^{2}\right) y_{\max }-b y_{\max }^{2}+\frac{y_{\max }^{3}}{3}\right]$

Similarly, for the obtained value of $y_{\max }$, the expression for PUIP can be written using equations (17) and (5) as follows (see [10] for derivations):

$$
\text { PUIP }=\int_{q=0}^{y_{\max }} \frac{2(b-q)}{\left(b^{2}-a^{2}\right)} \mathrm{d} q
$$

which can be solved to obtain

$$
P U I P=\frac{2 b y_{\max }-y_{\max }^{2}}{b^{2}-a^{2}}
$$

\section{Simulation Results and Performance EVALUATION}

We use OPNET simulator [11] to simulate the PU activity on an 11 Mbps wireless channel as Alternating Renewal Process, consisting of 10000 channel idle and 10000 channel busy periods that appear alternatingly. For Configuration-1, we use the channel idle and busy time distribution parameters as $\lambda_{i}=200 \mathrm{sec}^{-1}$ and $\lambda_{b}=500 \mathrm{sec}^{-1}$ respectively, with the mean values of 0.01 and 0.004 seconds respectively. For Configuration-2, we use the channel idle time distribution parameters $(a, b)=(0,2.0 \mathrm{sec})$, and busy time distribution parameters $(c, d)=(0,0.8 \mathrm{sec})$ with the mean values of 1.0 and 0.4 seconds respectively. For each value of the acceptable PU interference constraint $(\eta)$, using simulation we obtain the average channel utilization by SN per white space, and the probability of interference to PU due to SN transmissions.

We consider four different SN traffic profiles: one saturated SN traffic profile, and three bursty SN traffic profiles, which are modeled using 2-state Markov chain with ON and OFF states. We represent the mean sojourn time for ON and OFF states as $\mu_{O N}$ and $\mu_{O F F}$ respectively. Parameter values for the three bursty SN traffic profiles for Configuration-1 and Configuration-2 are shown in Table I. The performance parameters are also calculated (for the respective $\eta$ values) using analytical expressions given in (13) and (14) (for Configuration 1), and (20) and (21) (for Configuration 2). The average channel utilization by SN per white space is computed using the maximum transmission opportunity the $\mathrm{SN}$ gets in each white space.

\section{A. Results}

Figure 4 and Figure 5 compare (for Configuration 1) the analytically estimated and simulation-based performance parameters for different PU interference constraints $(\eta)$ and for different SN traffic profiles. Figure 6 and Figure 7 show these values for Configuration 2. We make several observations from these figures. First, the larger the value of $\eta$ (i.e. larger acceptable PU interference), the higher the channel utilization by SN per white space. This is so because the analytical formulations ((10) and (19)) predict larger transmission times (i.e larger $y_{\max }$ values) and therefore, $\mathrm{SN}$ transmits aggressively in each white space. However, such aggressive transmission by the SN leads to higher PU interference. For smaller values of $\eta$, the estimated values of $y_{\max }$, and consequently the SN transmissions, are conservative (so that it can meet the low interference requirement of the Primary Network), which leads to low white space utilization by the SN but low interference to the PU. Second, the analytically computed value PUIP serves as an upper bound for probability of interference to PU's transmission (due to secondary transmissions). The proposed theory ensures that the acceptable PU interference bound is never violated due to $\mathrm{SN}$ transmissions. The analytically computed $A U P W S$ values matches reasonably well with the simulation-obtained values for saturated SN traffic. For SN traffic profiles with low duty cycle (or low burstiness), such as Profile 1, the SN does not have enough frames to consume the estimated maximum transmission duration $y_{\max }$ in the remaining white space. As the the burstiness of SN traffic increases (from Profile 1 to Profile 3), the values of both of these performance parameters increase, and approaches the saturated SN traffic case.

We compare our proposed scheme with a Listen-BeforeTalk (LBT) scheme, which senses the channel for every frame transmission till it either collides with the PU transmission or senses the channel busy (due to PU transmission). In both these cases, the SN backs off exponentially with mean channel busy time parameter, and senses the channel again. We compare two performance parameters: average throughput of the Secondary Node (total number of SN frames transmitted / total simulation time) and the number of $S N$ frames transmitted per sensing operation (total number of SN frames transmitted / total number of sensing operations performed). Since single frame transmission scheme of LBT does not comply with any PUspecified interference constraint, in simulations, we observe the PU interference generated by the LBT scheme and then run our proposed scheme for the same value of the PU interference constraint. This enable us to compare the two schemes for similar PU interference constraints. Figures 8 and Figure 9 show respectively the average $S N$ throughput and $S N$ frames transmitted per sensing operation for LBT and the residual 


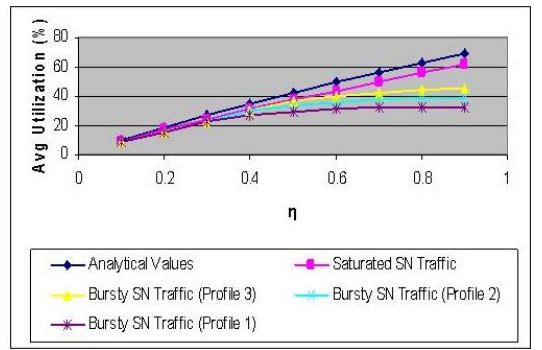

Fig. 4: Avg Utilization per White Space for Configuration 1 (Comparison of Analytical and Simulation Values); SN Frame Size $=2048$ bits

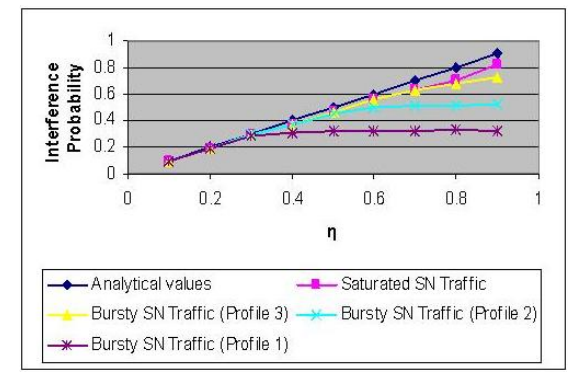

Fig. 7: PU Interference Probability for Configuration 2 (Comparison of Analytical and Simulation Values); SN Frame Size $=2048$ bits

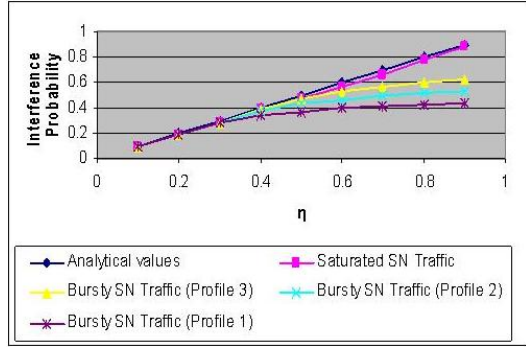

Fig. 5: PU Interference Probability for Configuration 1 (Comparison of Analytical and Simulation Values); SN Frame Size $=2048$ bits

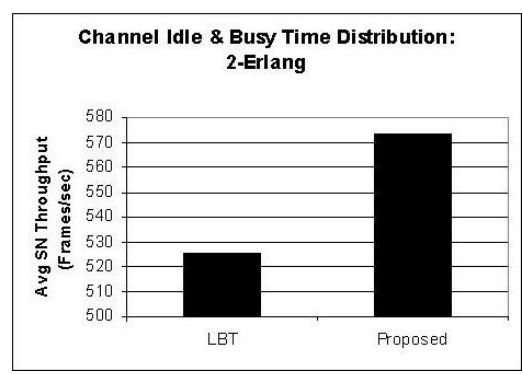

Fig. 8: Average SN Throughput for LBT and the Proposed Scheme $\left(I \sim 2-\operatorname{Erlang}\left(\lambda_{i}=\right.\right.$ $\left.200 \mathrm{sec}^{-1}\right) ; B \sim 2$-Erlang $\left(\lambda_{b}=500 \mathrm{sec}^{-1}\right)$; $\mathrm{SN}$ Frame Size $=1$ KBytes)

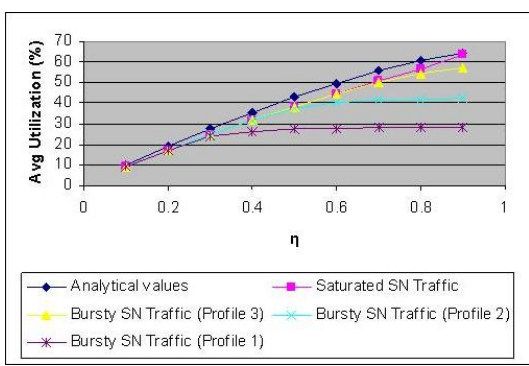

Fig. 6: Avg Utilization per White Space for Configuration 2 (Comparison of Analytical and Simulation Values); SN Frame Size $=2048$ bits

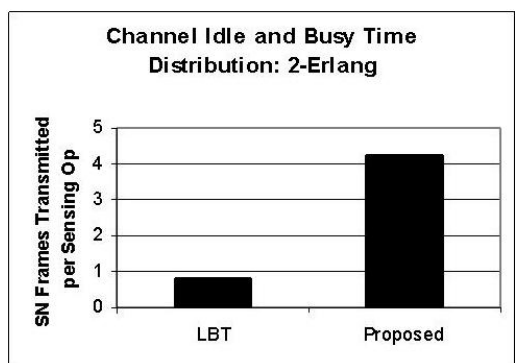

Fig. 9: Avg Secondary Frames Transmitted per Sensing Operation for LBT and the Proposed Scheme $\left(I \sim 2-\operatorname{Erlang}\left(\lambda_{i}=\right.\right.$ $\left.200 \sec ^{-1}\right) ; B \sim 2$-Erlang $\left(\lambda_{b}=500 \sec ^{-1}\right)$; SN Frame Size $=1$ KBytes)

\section{REFERENCES}

[1] Q. Zhao, L. Tong, A. Swami, and Y. Chen, "Decentralized cognitive MAC for opportunistic spectrum access in ad hoc networks: A POMDP framework," IEEE Jornal on Selected Areas in Communications: Special Issue on Adaptive, Spectrum Agile and Cognitive Wireless Networks, vol. 25, no. 3, April 2007.

[2] A. T. Hoang, Y. C. Ling, D. Yong, Y. Zeng, and R. Zhang, "Opportunistic Spectrum Access for Energy-Constrained Cognitive Radios," IEEE Transactions on Wireless Communications, vol. 8, no. 3, March 2009.

[3] Q. Zhao and K. Liu, "Detecting, Tracking, and Expoiting Spectrum Opportunities in Unslotted Primary Systems," in Proceedings of IEEE Radio and Wireless Symposium (RWS), 2008.

[4] S. Shetty, M. Song, C. Xin, and E. K. Park, "A Learning-based Multiuser Opportunistic Spectrum Access Approach in Unslotted Primary Networks," in Proceedings of IEEE INFOCOM, 2009.

[5] S. Geirhofer, L. Tong, and B. M. Sadler, "Cognitive Medium Access: Constraining Interference Based on Experimental Models," IEEE Journal on Selected Areas in Communications, vol. 26, no. 1, January 2008.

[6] S. Huang, X. Liu, and Z. Ding, "Optimal Sensing-Transmission Structure for Dynamic Spectrum Access," in Proceedings of IEEE INFOCOM 2009, 2009.

[7] P. A. K. Acharya, S. Singh, and H. Zheng, "Reliable Open Spectrum Communications Through Proactive Spectrum Access," in IEEE TAPAS, Boston MA, August 2006.

[8] A. Plummer, M. Taghizadeh, and S. Biswas, "Measurement Based Capacity Scavenging via Whitespace Modeling in Wireless Networks," in Proc. of IEEE GLOBECOM, 2009.

[9] K. S. Trivedi, Probability and Statistics with Reliability, Queuing and Computer Science Applications, 2nd ed. John Wiley and Sons, Inc., 2002.

[10] M. Sharma and A. Sahoo, "Residual White Space Distribution Based Opportunistic Channel Access Scheme for Cognitive Radio Ssystems," Indian Institute of Technology (IIT) Bombay, Tech. Rep., March 2010. [Online]. Available: http://www.cse.iitb.ac.in/internal/techreports/reports/TR-CSE2010-30.pdf

[11] "Opnet network simulator," http://www.opnet.com. We also plan to extend the proposed scheme to multi SN and multi channel scenario, and to study the impact of the proposed MAC scheme on PU performance at the application level. 